\title{
DETECTION AND POSITION METHOD OF APPLE TREE IMAGE
}

\author{
Wenhua Mao ${ }^{1,2^{*}}$, Baoping Jia ${ }^{1}$, Xiaochao Zhang ${ }^{2}$, Xiaoan Hub ${ }^{2}$ \\ ${ }^{1}$ College of Food Science \& Nutritional Engineering, China Agricultural University, Beijing, \\ P. R. China 100086 \\ ${ }^{2}$ Institute of Mechatronis Technology and Application, Chinese Academy of Agricultural \\ Mechanization Sciences, Beijing, P. R. China 100083 \\ * Corresponding author, Address: Institute of Mechatronis Technology and Application, \\ Chinese Academy of Agricultural Mechanization Sciences, Beijing 100083, P. R. China, \\ Tel: +86-10-64882667,Fax:+86-10-64882652,Email:mwh-924@163.com
}

Abstract: Apples should be quickly and correctly detected from their surroundings for the apple harvesting robot. The basic color feature was extracted from FuJi apple tree images and analyzed by the statistical analysis method. Accordingly, a new apple detection method was proposed to position the centroid of picking apples. The color difference was used to segment apples from their surroundings. Then the picking apples were chosen by area parameter. After that, the conglutinated apples were segmented by bidirectional scanning line algorithm. Finally, all of picking apples were positioned by their circumdiameter matching algorithm. The experimental result showed that the correct classification rate of apple fruit achieved $90 \%$.

Keywords: apple detection, image processing, color difference, position

\section{INTRODUCTION}

An apple harvesting robot must have the ability to segment an apple from its surroundings. Machine vision is an available method by which to capture images and to process color, shape and texture information. The past research on detecting fruit can be divided into two categories: local analysis (intensity and color information on the desired object) and shape analysis (fitting of circles or ellipses)( Jiménez et al.,2000, Plebe et al.,2001,

Please use the following format when citing this chapter:

Mao, W., Jia, B., Zhang, X. and Hub, X., 2009, in IFIP International Federation for Information Processing, Volume 294, Computer and Computing Technologies in Agriculture II, Volume 2, eds. D. Li, Z. Chunjiang, (Boston: Springer), pp. 1039-1048. 
Takahashi et al., 2002, Zhao et al.,2005). The shape analysis is more robust, while the local analysis is faster.

Bulanon et al. $(2001,2002)$ use luminance and color difference transformations of RGB color to recognize apple fruit in images. Stanjnko et al. (2004) apply thermal imaging to detect apples in the late afternoon for the purposes of calculating the fruit load. Zhao et al. (2005) used a combination of redness index $(r=3 R-(G+B))$, texture-based edge detection, and circle fitting in RGB color. Amy et al. (2006) developed a method for segmentation of apple fruit from video via background modeling. Global Mixture of Gaussians (GMOG), is based on the principles of Mixture of Gaussians (MOG), was used for motion-detection applications.

But the apple segmentation based on machine vision contains some avoidless factors. One of the main factors is variable lighting for the environment is outdoor. Another is that of occlusion, including occlusion of apples by leaves and branches of the tree, occlusion by other apples and by trellis poles and wires.

Therefore, the objectives of this research:

1.to develop a novel method for apple segmentation using the color feature to avoid the influence of variable lighting;

2.to develop a new method for position the picking apples and segmentation the occlusion of apple fruit by leaves, branches, trellis poles, and even by other apple fruit.

\section{APPLES SEGMENTATION WITH COLOR FEATURE}

In general, the ripe Fuji apple is red, and the surrounding of apples is composed of green leaves and weeds, taupe branches, sky and soil, as shown in Fig.1. In order to segment apples from compound background, the software SPSS 13.0 for windows was used to find the distinct color feature of apples.

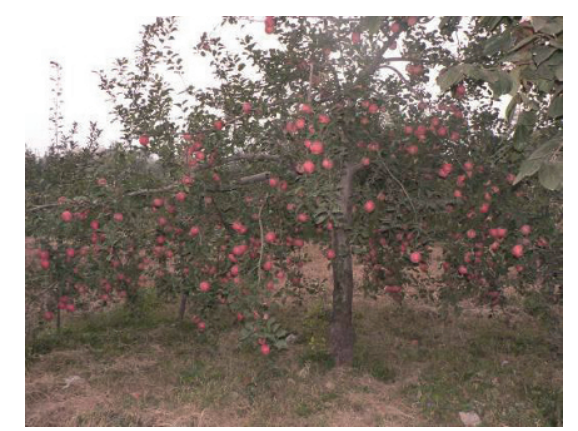

Fig .1: The captured image of Fuji apples tree 


\subsection{Color feature analysis}

50 frames color images of apple trees were taken by the use of a color digital camera (Panasonic DMC-FZ5) in outdoor natural lighting conditions during 2006/2007 in the Sino-Japanese friendship tour orchard. The captured images included various different conditions, such as the relative distance of camera and apple trees, the lighting (sunny or cloudy), the time (a.m. or p.m.).

25 frames chosen randomly from the 50 frames collected color images were used for the statistical analysis as the follow steps:

\section{Step1. Preprocess}

The original image included object (apples) and background (leaves, branches, sky, soil and weed). Their region of interesting were manually extracted from the source images to built the sub-image settings of apples, leaves, branches, sky ,soil and weed, respectively. Then, the RGB triplets of six classes were automatically acquired from the various sub-image settings. The number of captured data of each class was shown in the table 1 .

Table 1. The captured data of each class in the RGB color model

\begin{tabular}{|c|c|c|c|c|c|}
\hline \multirow{2}{*}{$\begin{array}{c}\text { Label } \\
\text { (apple) }\end{array}$} & \multicolumn{3}{|c|}{ Label (background) } & \multicolumn{2}{|c|}{4900} \\
\hline & branch & leaf & sky & soil & weed \\
\hline 980 & 980 & 980 & 980 & 980 & 980 \\
\hline
\end{tabular}

\section{Step2. RGB data Analysis}

In the RGB color model, the box plot of each class (Fig.2) was drawn. It could be seen from the Fig.2 that:

1) The $R$ index of apple was larger than the other classes'.

2 ) The $R$ index was larger than the $G$ and B indexes for apple and soil, but the $R$ index of apple was larger than the soil's.

3) The $B$ index of sky was larger than the other classes'.

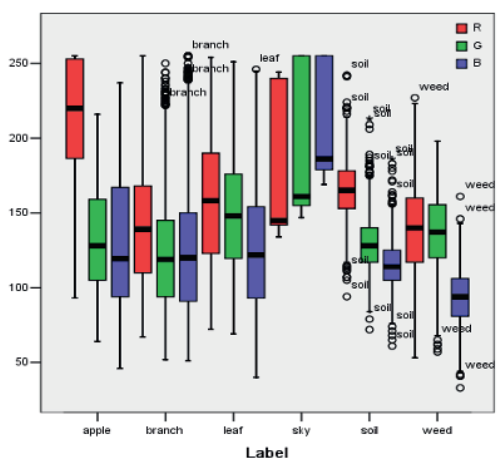

Fig .2: The box plot of six classes in the RGB triple 


\section{Step3. 2D data Analysis}

The RGB colors were transformed into $2 \mathrm{D}$ perspective chromaticity spaces that were mainly emphasized the $\mathrm{R}$ index and commonly used in computer vision:

1) the difference indexing:

$$
\begin{aligned}
& D_{r g}=R-G \\
& D_{r b}=R-B
\end{aligned}
$$

2) the HS indexing:

$$
\begin{array}{ll}
S=1-\frac{3}{R+G+B}[\min (R, G, B)] & \\
H= \begin{cases}\frac{\pi}{2}+\operatorname{arctg} \frac{\sqrt{3}(G-B)}{2 R-G-B}, & G \geq B \\
\frac{3 \pi}{2}-\operatorname{arctg} \frac{\sqrt{3}(G-B)}{2 R-G-B}, & G<B\end{cases}
\end{array}
$$

3) the ratio indexing:

$$
\begin{aligned}
& R_{r g}=\frac{R}{G} \\
& R_{r b}=\frac{R}{B}
\end{aligned}
$$

4) the normalization indexing:

$$
\begin{aligned}
& r=\frac{R}{R+G+B} \\
& g=\frac{G}{R+G+B}
\end{aligned}
$$

The scatter plots of those 2D perspective chromaticity spaces (Fig.3) were drawn as apple and background classes. It could be seen from them that: All of those 2D perspective chromaticity spaces could segment apples from background, excluding little of them for data occluding.

The $D_{r g}-D_{r b}, H-S, R_{r g}-R_{r b}$ and $r-g$ data set were used to make the discrimination classification for the selection of 2D color indexing of apple detection. The discriminant function calculated with the enter independents together method, that is based on the within-group covariance, on the assumption that prior probabilities of all groups were equal. The result was shown in the table 2. The correct classification rate (abbr. CCR) of $D_{r g}-D_{r b}$ color indexing was higher than others. Therefore, the $D_{r g}-D_{r b}$ color indexing was optimal. 


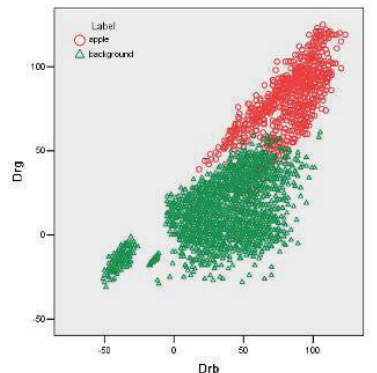

(a) $\mathrm{D}_{\mathrm{rg}}-\mathrm{D}_{\mathrm{rb}}$

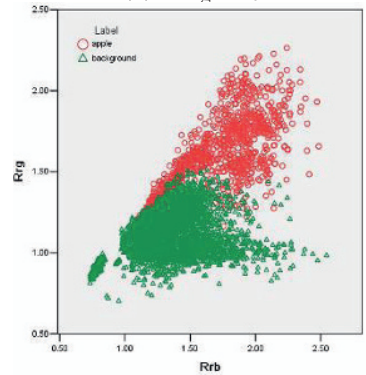

(c) $\mathrm{R}_{\mathrm{rg}}-\mathrm{R}_{\mathrm{rb}}$

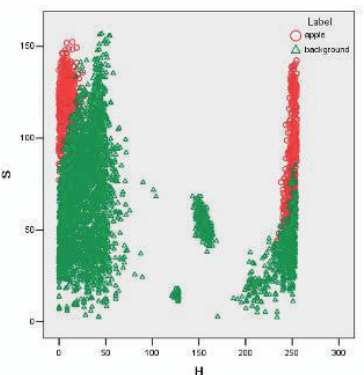

(b) H-S

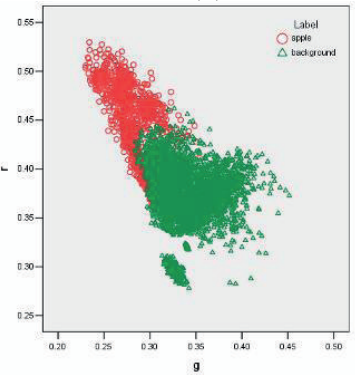

(d) $\mathrm{r}-\mathrm{g}$

Fig .3: The scatter plots of apple and background classes

Table 2. The CCR of apple and background in 2D color indexing

\begin{tabular}{lll}
\hline Color indexing & CCR of apple & CCR of background \\
\hline$D_{r g}-D_{r b}$ & $93.8 \%$ & $98.2 \%$ \\
$H-S$ & $92.2 \%$ & $86.7 \%$ \\
$R_{r g}-R_{r b}$ & $89.7 \%$ & $97.1 \%$ \\
$r-g$ & $93.1 \%$ & $94.2 \%$ \\
\hline
\end{tabular}

\subsection{Segmentation method in optimal color indexing}

The nearest neighbor clustering method was used to segment apples from background in $D_{r g}-D_{r b}$ color indexing. If the distance of a pixel point $P\left(D_{r g}\right.$, $\left.D_{r b}\right)$ with the apple's initial point $\mathrm{P}\left(D a_{r g}, D a_{r b}\right)$ was less than the distance with the background's initial point $P\left(D b_{r g}, D b_{r b}\right), P\left(D_{r g}, D_{r b}\right)$ was the apple class; on the contrary, $P\left(D_{r g}, D_{r b}\right)$ was the background class.

If $\left\|P\left(D_{r g}, D_{r b}\right)-P\left(D a_{r g}, D a_{r b}\right)\right\|<\left\|P\left(D_{r g}, D_{r b}\right)-P\left(D b_{r g}, D b_{r b}\right)\right\|$, $P\left(D_{r g}, D_{r b}\right) \in$ Apple ; else $P\left(D_{r g}, D_{r b}\right) \in$ Background .

Where, $P\left(D a_{r g}, D a_{r b}\right)$ and $P\left(D b_{r g}, D b_{r b}\right)$ were set the median of $D_{r g}-D_{r b}$ color indexing of apple and background classes, respectively:

$$
\begin{aligned}
& P\left(D a_{r g}, D a_{r b}\right)=P(82,89) \\
& P\left(D b_{r g}, D b_{r b}\right)=P(10,26)
\end{aligned}
$$




\section{APPLES POSITION WITH SHAPE FEATURE}

After the source image was segmented by the $D_{r g}-D_{r b}$ color indexing, the picking apples were positioned as follow flowchart (Fig.4):

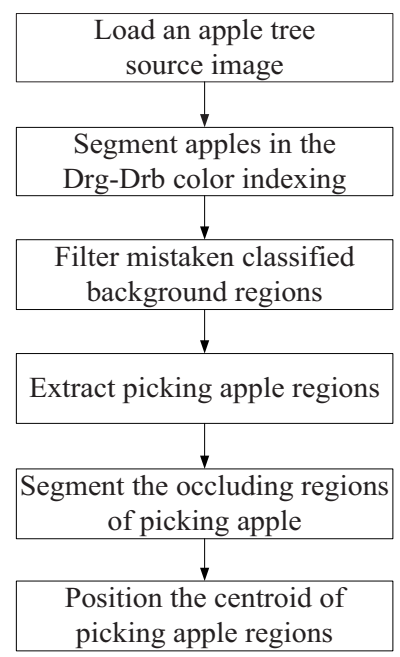

Fig.4: The flowchart of an apple tree image processing

\subsection{Filter background noise}

The segmentation image was composed of most apples and little background pixels. The area of apple region was much larger than the area of background region. Therefore, the mini-area erasion algorithm was used to filter the background noise.

All object regions were fast labeled and their areas were computed. The area threshold of erasing background region $\left(T_{b}\right)$ was equal to the mean area of all object regions $\left(A_{m}\right)$. If the area of a region was less than $T_{b}$, this region was erased from object regions, and its area data also was excluded.

\subsection{Extract picking apples}

If an apple had a near distance to the harvesting robot, its area was correspondingly larger and less occluding. Therefore, the picking apples were extracted as follow:

1)The edge of apple regions was smoothed by the opening and closing algorithm. The holes in apple region were filled by the hole filling algorithm.

2)The area threshold of picking apple (Ta) was equal to the mean area of all apple regions (Ama). If the area of a region was less than $\mathrm{Ta}$, this region was an unpicking apple, and it was filtered also from object regions. 


\subsection{Segment occluding apples}

An apple may be occluded by leaves, branches, trellis poles, and even by other apple fruit. And the occluding scale, orientation and shape were difference in thousand ways. But the segmentation line of occluding parts had some variation points. The pixel value of a variation point was 0 , and the pixel values of left and right points connected with it were 255 .

Therefore, the bidirectional scanning line algorithm was used to segment occluding apple regions, which were scanned from the horizontal and vertical direction (Fig.5). If there were three variation points in a scanning line, and all of them were not start or end pixels $(D(x, y))$, then the second variation point was a occluding point of two objects $(C(x, y))$. The segmentation line of two objects was the line connected with two $C(x, y)$.

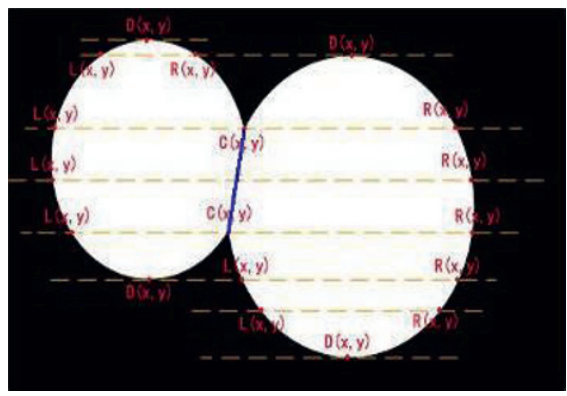

(a) horizontal scanning

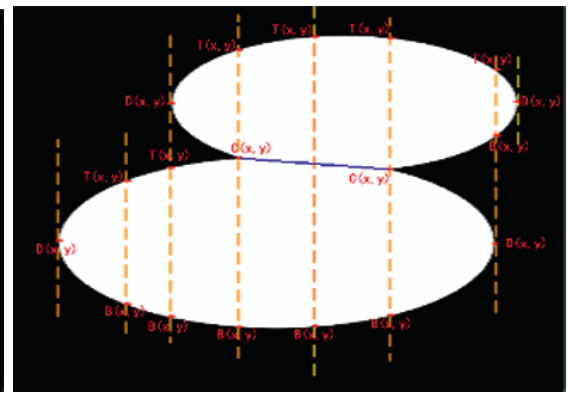

(b) vertical scanning

Fig.5: The sketch map of the bidirectional scanning line algorithm:

Similarly, the segmented regions of unpicking apples were filtered by the extract picking apples algorithm.

\subsection{Position the centroid of picking apples}

The shape feature of single apples was similar, they were approximate circle. Therefore, the centroid of picking apple was positioned by the shape feature as follows:

1) The Roundness of picking apples $(R)$ were computed as the followed formula:

$$
R=\frac{4 A}{\pi L^{2}}
$$

Where, $\mathrm{A}$ and $\mathrm{L}$ were the area and length of object, respectively.

The single picking apples were selected by the $\mathrm{R}$ : if $0.9<\mathrm{R}<1$, then object was a rounding apple; on the contrary, object only was a missing apple.

The centroid of rounding apple(xc,yc)was positioned as the formula: 


$$
\begin{aligned}
& x_{c}=\frac{1}{A} \sum_{(x, y \in R)} x \\
& y_{c}=\frac{1}{A} \sum_{(x, y \in R)} y
\end{aligned}
$$

2) The centroid of missing apple was positioned by the circum-diameter matching algorithm. The circum-diameter $\left(D_{c}\right)$ was computed as the formula:

$$
D_{c}=2 \times \max \left(\left\|P(x, y)-P\left(x_{c}, y_{c}\right)\right\|\right)
$$

Where, $P(x, y)$ was an edge point of object. If the length of two edge points was furthest matching with the $D_{c}$, this line was the central axis of apple. Its' midpoint was the centroid of missing apple.

\section{TEST AND RESULT}

10 frames of test images with shading and brightness regions were randomly chosen from the 50 frames collected color images, for testing the detection effect and position precision. The segmentation result of an image was shown in the Fig.6. The apple pixels were white, and the background pixels were black.

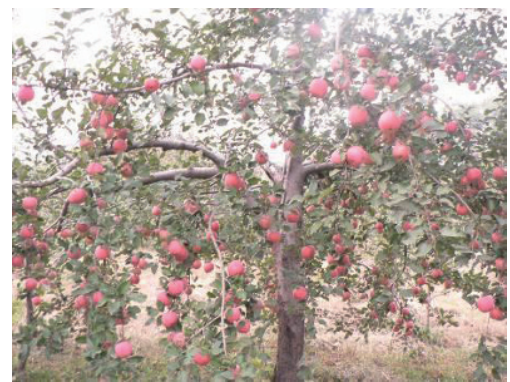

(a) source image

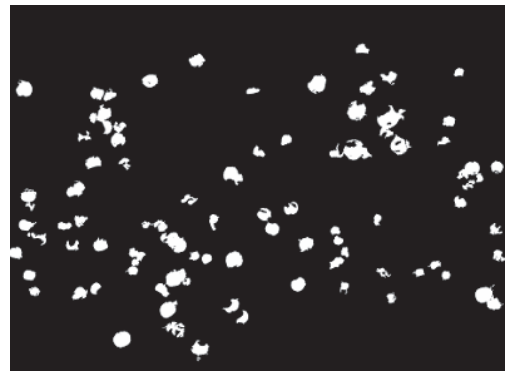

(c) filter background noise

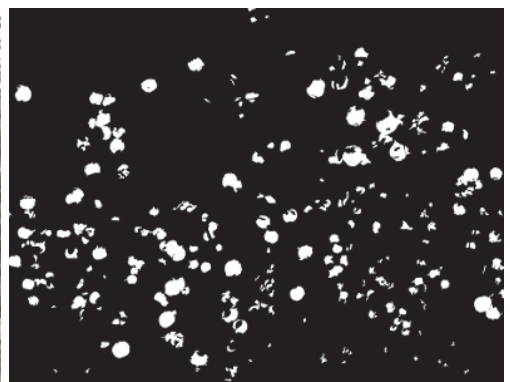

(b) segmented background

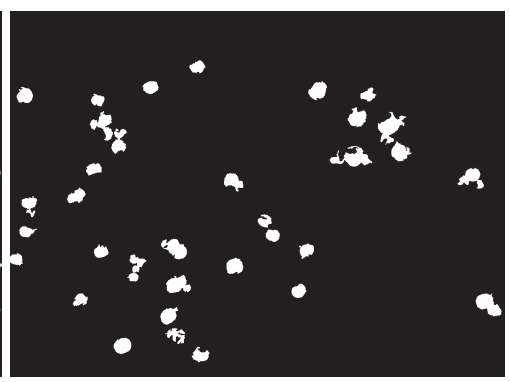

(d) extract picking apples 


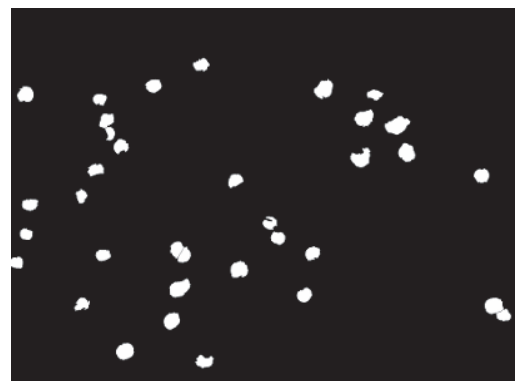

(e) segment occluding apples

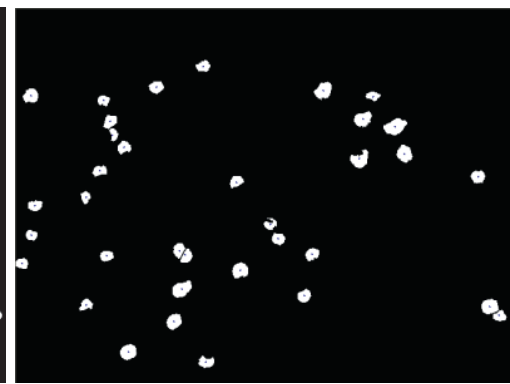

(f) position the centroid

Fig.6: The result images processed step by step

The result of image processing was compared with the result of eye detection (Table 3). The number of picking apple detected by image processing (abbr. IP) ( $\mathrm{Na}$ ) was automatically gained in the above mentioned algorithm. The number of picking apple detected by eye $(\mathrm{Ne})$ was manually counted from the source image. The Error $(E)$ was computed by the follow formula:

$$
E=\frac{\left|N_{a}-N_{e}\right|}{N_{e}} \times 100 \%
$$

Table 3. The compare of image processing and eye detection

\begin{tabular}{cccc}
\hline Image & $\begin{array}{c}\text { Number of picking apple } \\
\text { detected by IP }\end{array}$ & $\begin{array}{c}\text { Number of picking apple } \\
\text { detected by eye }\end{array}$ & $\begin{array}{c}\text { Error } \\
(\%)\end{array}$ \\
\hline 1 & 35 & 32 & 9.4 \\
2 & 28 & 29 & 3.4 \\
3 & 24 & 23 & 4.3 \\
4 & 31 & 30 & 3.3 \\
5 & 37 & 34 & 8.8 \\
6 & 24 & 26 & 7.7 \\
7 & 20 & 22 & 9.1 \\
8 & 26 & 24 & 8.3 \\
9 & 30 & 33 & 9.1 \\
10 & 18 & 19 & 5.3 \\
\hline
\end{tabular}

\section{CONCLUSION}

1. Develop an optimal $D_{r g}-D_{r b}$ color indexing for segmentation apples from background;

2. Develop a bidirectional scanning line algorithm for segmentation the occlusion apples;

3. Develop a circum-diameter matching algorithm for position the centroid of occlusion apples.

4. Get the data of number of picking apples with a maximum error $9.4 \%$. 


\section{ACKNOWLEDGEMENTS}

Funding for this research was provided by the Postdoctors Fund in China and the Ministry of Science and Technology of the P.R.China. The first author is grateful to the China Agricultural University for providing her with pursuing a postdoctor work.

\section{REFERENCES}

A. Plebe, G. Grasso. Localization of spherical fruits for robotic harvesting, Machine Vision and Applications,2001, 13 (2): 70-79

A. R. Jiménez, R. Ceres, J. L. Pons. A survey of computer vision methods for locating fruit on trees, Trans. ASAE, 2000, 43(6): 1911-1920

D. M. Bulanon, T. Kataoka, S. Zhang, Y. Ota, T. Hiroma. Optimal thresholding for the automatic recognition of apple fruits, ASAE Paper, 2001,No. 01-3133

D. M. Bulanon, T. Kataoka, Y. Ota, and T. Hiroma. A Color Model for Recognition of Apples by a Robotic Harvesting System,Journal of the JSAM, 2002, 64(5):123-133

D. Stanjnko, M. Lakota, M. Hocevar. Estimation of number and diameter of apple fruits in an orchard during the growing season by thermal imaging, Computers and Electronics in Agr, 2004,42: 31-42

J.Zhao, J. Tow, J. Katupitiya. On-tree fruit recognition using texture properties and color data,IEEE/RSJ Int. Conf. Intell. Robots and Systems, 2005,263-268

L. T. Amy, L. P.Donald, P. Johnny. Segmentation of Apple Fruit from Video via Background Modeling, ASAE Paper, 2006, No. 063060

T. Takahashi, S. Zhang, H. Fukuchi. Measurement of 3-D Locations of Fruit by Binocular Stereo Vision for Apple Harvesting in an Orchard, ASAE Paper, 2002, No. 021102 